\title{
Effect of androgen on bone metabolism in hyperuricemic rats
}

Li Ling ${ }^{1}$, Yun-Xiao Wei², Yi-Fei Sun², Meng-Jiao Zhang², Jie Chen², Shi-Ying Luo², Jin-Wei Xue ${ }^{3}$

\begin{abstract}
${ }^{1}$ Department of Neurology, Affiliated Hospital of Hebei University, Baoding, Hebei, China ${ }^{2}$ Department of Neurology, Hebei University, Baoding, Hebei, China

${ }^{3}$ Department of Orthopedics, Affiliated Hospital of Hebei University, Baoding, Hebei, China
\end{abstract}

Submitted: 20 March 2019

Accepted: 8 September 2019

Arch Med Sci

DOI: https://doi.org/10.5114/aoms.2020.96280

Copyright $\odot 2020$ Termedia \& Banach

\begin{abstract}
Introduction: This study aimed to investigate the effect of androgen on bone metabolism in hyperuricemic rats.

Material and methods: Forty male Wistar rats were randomly divided into four groups: sham operation group, simple hyperuricemic group, hyperuricemic castration group, and simple castration group. A rat model of chronic hyperuricemia was established using potassium oxonate and ethambutol. Blood was sampled from the vena angularis at week 0, 4, 6, 8 and 12 after surgery to detect for uric acid, calcium, phosphorus and alkaline phosphatase, and investigate the effect of androgen on bone metabolism in hyperuricemic rats.
\end{abstract}

Results: From the $4^{\text {th }}$ week, compared with the sham operation group, the differences in uric acid levels between the simple hyperuricemic group and hyperuricemia castration group were statistically significant $(p<0.05)$, suggesting the successful establishment of the model of hyperuricemia. At the $6^{\text {th }}$ week, uric acid levels decreased in the two hyperuricemic groups, and the difference from the sham operation group decreased $(p=0.05)$, showing that the modeling method had deteriorated. At the $8^{\text {th }}$ week, the differences in uric acid levels between the two castration groups and sham operation group were statistically significant $(p<0.05)$. At the $12^{\text {th }}$ week, the differences in serum levels of phosphorus between the simple hyperuricemic group and hyperuricemic castration group were statistically significant $(p<0.05)$.

Conclusions: Androgen can induce bone metabolism changes in rats with hyperuricemia.

Key words: hyperuricemia, androgens, bone metabolism.

\section{Introduction}

With the aging of the population and changes in lifestyle, the prevalence of bone diseases and metabolic diseases continues to increase [1], in which hyperuricemia and osteoporosis are especially significant [2]. Osteoporosis is associated with high oxidative stress levels and weak antioxidant barriers $[3,4]$. The increase in osteoclastic activity detected in osteoporosis may be responsible for the increase in reactive oxygen species (ROS) production [3]. Serum uric acid is the end product of purine metabolism, and can be detrimental in many metabolic [5]

\author{
Corresponding author: \\ Jin-Wei Xue \\ Department of Orthopedics \\ Affiliated Hospital of Hebei \\ University \\ Baoding, Hebei, China \\ E-mail: \\ xuejw_ww66@163.com
}


and cardiovascular disorders [6]. Serum uric acid accounts for approximately $50 \%$ of extracellular antioxidant activity [7]. Some recent studies have shown that higher serum uric acid levels are positively correlated with biomarkers of better bone health, leading to speculation about the potential protective role of uric acid against bone loss [8-10]. However, urate crystals promoted osteoclast growth and reduced osteoblast activity, suggesting the globally negative effect of uric acid on bone metabolism [11]. Furthermore, other studies have revealed that hyperuricemic status is correlated with osteoporosis [12-14]. Androgen is known to play a very important role in bone metabolism. Anabolic sex hormones are necessary for bone matrix synthesis, and the loss of androgens with aging in men causes osteoporosis [15]. Furthermore, androgen can maintain the stability of bones [8, 16-18]. However, it remains to be determined whether androgen has an effect on bone and bone metabolism under hyperuricemic status. Therefore, the present study was designed to explore the effect of androgen on bone metabolism in hyperuricemic rats through animal experiments.

\section{Material and methods}

\section{Experimental materials}

\section{Main reagents}

Potassium oxonate was produced by Shandong Zhongke Taidou Chemical Co. Ltd., with a purity of $>98 \%$. Adenine was produced by Shanghai ZZBIO Ltd., with a purity of $>95 \%$. The reagent was dissolved with $0.5 \%$ sodium carboxymethyl cellulose (CMC-Na) before use.

\section{Experimental animals}

All experimental procedures were performed according to the protocols approved by the Animal Ethical and Welfare Committee of Hebei University [No. IACUC-2017005]. The rats were provided by Beijing Vital River Laboratory Animal Technology Co., Ltd. Feeding and management conditions: Rats were kept in a specific-pathogen-free (SPF) animal laboratory, with a room temperature of $20 \pm 2^{\circ} \mathrm{C}$ and a relative humidity of $60-70 \%$, and maintained on a 12 -hour reverse light-dark cycle with ad libitum access to food and water. The weights of rats were measured once a week, and changes in the body weight of rats were monitored and recorded. Rats were given the corresponding drug dosage according to weight.

\section{Experimental methods}

\section{Establishment of the animal model}

Forty clean, healthy, male four-month-old Wistar rats, weighing 280-320 g, were used for the present study. After seven days of adaptive feeding in the laboratory, these rats were randomly divided into four groups using a random number table $(n=10$, each): sham operation group, simple hyperuricemic group, hyperuricemic castration group, and simple castration group.

1. Establishment of the castrated rat model: Rats in these two castration groups were treated with orchiectomy and epididymis resection, while the fat tissue around the testis in rats in the sham operation group was removed. These rats were defined as controls.

2. Establishment of the model of hyperuricemic rats $[19,20]$ : From 10 a.m. every day, rats in the simple hyperuricemic group were given $1.0 \mathrm{~g} /$ $\mathrm{kg}$ of potassium oxonate for continuous gastric lavage, while rats in the hyperuricemic castration group were treated with intragastric administration of $1.0 \mathrm{~g} / \mathrm{kg}$ of potassium and a subcutaneous injection of $100 \mathrm{mg} / \mathrm{kg}$ of potassium oxonate. Rats in other groups were treated by gavage using an equal volume of distilled water.

3. Adjustment of modeling methods in rats with hyperuricemia [20]: After the level of uric acid decreased in rats in the simple hyperuricemic group and hyperuricemic castration group, the method for the modeling was altered. Rats in the simple hyperuricemic group were continuously gavaged with $1.5 \mathrm{~g} / \mathrm{kg}$ of potassium oxonate daily, and subcutaneously injected with 200 mg/ $\mathrm{kg}$ of ethambutol, while rats in the hyperuricemic castration group were treated with the intragastric administration of $1.0 \mathrm{~g} / \mathrm{kg}$ of potassium oxonate, a subcutaneous injection of $100 \mathrm{mg} / \mathrm{kg}$ of potassium oxonate, and a subcutaneous injection of $200 \mathrm{mg} / \mathrm{kg}$ of ethambutol, for a total of 12 weeks.

\section{Blood sampling and testing}

Rats were fasted for 12 hours before each blood sampling. According to the body weight of rats, each rat was anesthetized via intraperitoneal injection of $3 \%$ pentobarbital sodium at a dose of $1.5 \mathrm{ml} / \mathrm{kg}$. Then, $2 \mathrm{ml}$ of blood was sampled from the vena angularis in each rat. Afterwards, these rats were continuously gavaged for 12 weeks.

Drug dosage was adjusted according to the body weight of rats, while the administration time and route were the same for rats in the different groups.

\section{Determination of serum uric acid and bone metabolism indexes}

Blood was sampled from the vena angularis at week $0,4,6,8$ and 12 , respectively, for the determination of uric acid, calcium, phosphorus and alkaline phosphatase. 


\section{Statistical analysis}

Data were statistically analyzed using the statistical software SPSS 19.0. Measurement data were expressed as mean \pm standard deviation $(x \pm S D)$. Data were compared using univariate analysis of variance.

\section{Results}

\section{General changes in experimental rats}

During the experiment, the body weight of rats in the two hyperuricemic groups initially increased, and subsequently decreased. Furthermore, rats in these two groups had listlessness, dry and yel- low hair, and reduced movements, while rats in the control group were smart and active, and had shiny body hair and weight gain. Furthermore, rats in each group were given ad libitum access to food and water during the experiment. During the experiment, four rats died in the simple hyperuricemic group and hyperuricemic castration group.

\section{Uric acid and bone metabolism indexes} of rats in each group (Tables I-V)

Serum uric acid levels gradually increased at week four after the establishment of the model, and the difference was statistically significant $(p<0.05$, Table II). During this period, the model

Table I. Uric acid and bone metabolism indexes of rats in each group before the experiment $(n=40, \mathrm{x} \pm \mathrm{s}, \mathrm{mmol} / \mathrm{l})$

\begin{tabular}{|lcccc|}
\hline Group & UA & Ca & P & ALP \\
\hline Sham operation group & $90.94 \pm 11.15$ & $2.57 \pm 0.06$ & $2.30 \pm 0.22$ & $177.31 \pm 34.24$ \\
\hline Simple hyperuricemia group & $100.97 \pm 24.18$ & $2.54 \pm 0.09$ & $2.23 \pm 0.15$ & $171.21 \pm 27.36$ \\
\hline Hyperuricemia castration group & $97.75 \pm 22.12$ & $2.54 \pm 0.09$ & $2.16 \pm 0.23$ & $168.91 \pm 34.14$ \\
\hline Simple castration group & $99.39 \pm 15.50$ & $2.55 \pm 0.10$ & $2.15 \pm 0.22$ & $166.21 \pm 41.20$ \\
\hline
\end{tabular}

UA - uric acid, Ca - calcium, $P$ - phosphorus, ALP - alkaline phosphatase. There was no difference in serum indexes between each group.

Table II. Uric acid and bone metabolism indexes of rats in each group at the $4^{\text {th }}$ week $(n=40, x \pm s, \mathrm{mmol} / \mathrm{l})$

\begin{tabular}{|lcccc|}
\hline Group & UA & Ca & P & ALP \\
\hline Sham operation group & $106.69 \pm 27.16$ & $2.51 \pm 0.06$ & $2.37 \pm 0.05$ & $165.34 \pm 16.17$ \\
\hline Simple hyperuricemia group & $140.60 \pm 123.97^{a}$ & $2.48 \pm 0.10$ & $2.39 \pm 0.10$ & $159.15 \pm 27.94$ \\
\hline Hyperuricemia castration group & $135.77 \pm 23.65^{a}$ & $2.44 \pm 0.09$ & $2.27 \pm 0.12$ & $154.62 \pm 29.80$ \\
\hline Simple castration group & $104.09 \pm 12.12$ & $2.48 \pm 0.08$ & $2.33 \pm 0.13$ & $154.43 \pm 16.75$ \\
\hline
\end{tabular}

UA - uric acid, Ca - calcium, $P$ - phosphorus, ALP - alkaline phosphatase. ${ }^{a}$ Compared with sham operation group, $p<0.05$.

Table III. Uric acid and bone metabolism indexes of rats in each group at the $6^{\text {th }}$ week $(n=40, x \pm \mathrm{s}, \mathrm{mmol} / \mathrm{l})$

\begin{tabular}{|lcccc|}
\hline Group & UA & Ca & P & ALP \\
\hline Sham operation group & $108.91 \pm 20.16$ & $2.40 \pm 0.13$ & $2.33 \pm 0.38$ & $164.11 \pm 26.78$ \\
\hline Simple hyperuricemia group & $136.72 \pm 22.24^{\mathrm{a}, \mathrm{b}}$ & $2.13 \pm 0.19^{\mathrm{a}, \mathrm{b}}$ & $2.33 \pm 0.23$ & $134.50 \pm 18.79$ \\
\hline Hyperuricemia castration group & $135.36 \pm 24.14^{\mathrm{b}}$ & $2.11 \pm 0.21^{\mathrm{a}, \mathrm{b}}$ & $2.23 \pm 0.16$ & $137.40 \pm 25.31$ \\
\hline Simple castration group & $99.69 \pm 9.81$ & $2.28 \pm 0.30$ & $2.42 \pm 0.19$ & $137.15 \pm 16.92$ \\
\hline
\end{tabular}

UA - uric acid, Ca - calcium, $P$ - phosphorus, ALP - alkaline phosphatase. ${ }^{a}$ Compared with sham operation group, $p \leq 0.05 .{ }^{b} \mathrm{Compared}$ with simple castration group, $p<0.05$.

Table IV. Uric acid and bone metabolism indexes of rats in each group at the $8^{\text {th }}$ week $(n=39, \mathrm{x} \pm \mathrm{s}, \mathrm{mmol} / \mathrm{l})$

\begin{tabular}{|lcccc|}
\hline Group & UA & Ca & P & ALP \\
\hline Sham operation group & $118.10 \pm 20.32$ & $2.41 \pm 0.10$ & $2.27 \pm 0.23$ & $157.91 \pm 9.53$ \\
\hline Simple hyperuricemia group & $157.22 \pm 25.38^{\mathrm{ab}}$ & $2.11 \pm 0.13^{\mathrm{a}}$ & $2.17 \pm 0.17$ & $137.53 \pm 9.00$ \\
\hline Hyperuricemia castration group & $140.15 \pm 15.00^{\mathrm{ab}}$ & $2.06 \pm 0.19^{\mathrm{a}}$ & $2.10 \pm 0.18$ & $130.39 \pm 11.03$ \\
\hline Simple castration group & $104.58 \pm 12.35$ & $2.21 \pm 0.21^{\mathrm{a}}$ & $2.26 \pm 0.11$ & $138.22 \pm 16.35$ \\
\hline
\end{tabular}

UA - uric acid, Ca - calcium, $P$ - phosphorus, ALP - alkaline phosphatase. ${ }^{a}$ Compared with sham operation group, $p \leq 0.05 .{ }^{b}$ Compared with simple castration group, $p<0.05$. 
Table V. Uric acid and bone metabolism indexes of rats in each group at the $12^{\text {th }}$ week $(n=36, \mathrm{x} \pm \mathrm{s}, \mathrm{mmol} / \mathrm{l})$

\begin{tabular}{|c|c|c|c|c|}
\hline Group & UA & $\mathrm{Ca}$ & $P$ & ALP \\
\hline Sham operation group & $129.41 \pm 18.83$ & $2.41 \pm 0.17$ & $2.22 \pm 0.19$ & $149.18 \pm 10.44$ \\
\hline Simple hyperuricemia group & $172.01 \pm 21.32^{\mathrm{a}, \mathrm{b}}$ & $2.11 \pm 0.16^{a}$ & $2.14 \pm 0.14$ & $143.85 \pm 12.14$ \\
\hline Hyperuricemia castration group & $153.83 \pm 31.87^{a, b}$ & $2.06 \pm 0.17^{a}$ & $1.96 \pm 0.13^{\mathrm{a}, \mathrm{b}, \mathrm{c}}$ & $123.16 \pm 18.10^{a}$ \\
\hline Simple castration group & $106.59 \pm 10.71^{a}$ & $2.21 \pm 0.22^{\mathrm{a}}$ & $2.26 \pm 0.20$ & $131.15 \pm 18.17^{a}$ \\
\hline
\end{tabular}

of hyperuricemia was successfully established. However, there was no difference in calcium, phosphorus and alkaline phosphatase among all groups, which was possibly due to the short time of the model establishment.

At week six, uric acid level decreased in the two hyperuricemic groups, and the difference in uric acid levels decreased, and was not statistically significant $(p=0.05)$.

After week six, the modeling method was improved. The difference in calcium level in the hyperuricemic castration group was statistically significant, when compared with the castration groups ( $p=0.05$, Table IV) at week eight. Compared with the castration groups, the difference was statistically significant, indicating that hyperuricemia induced changes in bone metabolism. During this period, the differences in uric acid level among the sham operation group, simple hyperuricemic group and hyperuricemic castration group were statistically significant $(p<0.05)$. This indicates that the model of hyperuricemia was successful. One rat died in the hyperuricemic castration group, and the cause might be the persistent elevation in uric acid level.

At week 12, the differences in bone metabolism indexes, such as calcium, phosphorus and alkaline phosphatase in rats, were statistically significant ( $p<0.05$, Table V). During this period, one rat died in the simple hyperuricemic group, while another rat died in the hyperuricemic castration group. In total, four rats died and 36 rats survived, and the reason was considered to be the long-term hyperuricemia in rats. The difference in bone metabolism indexes between the simple hyperuricemic group and hyperuricemic castration group was statistically significant, indicating the effect of androgen on the bone metabolism in hyperuricemic rats.

\section{Discussion}

Osteoporosis is a disease characterized by bone fragility and increased risk of fracture [9, 21, 22]. The pathogenesis of osteoporosis is very complex, and involves genetic, lifestyle and environmental factors, as well as other important factors. Andro- gen plays an important role in bone growth, metabolism, bone mass maintenance and anti-bone loss [23]. In addition, the level of serum uric acid is also affected by androgen. Androgen inhibits uric acid excretion and increases uric acid production [24]. Although uric acid itself can also induce bone damage, the mechanism may be the changes in renal function induced by uric acid crystallization and the rich protein diet taken by hyperuricemic subjects, which leads to a decrease in calcium absorption [25]. In recent years, there has been a view [1] that the occurrence of osteoporosis is correlated with the disorder of differentiation of bone marrow mesenchymal stem cells. That is, bone marrow mesenchymal stem cells excessively differentiate into adipocytes in patients with osteoporosis, which is accompanied by the weakening of osteogenic differentiation ability and bone loss. This is an important cause of osteoporosis. Bone erosion is a common manifestation of chronic tophaceous gout. Monosodium urate monohydrate (MSU) crystals have profound inhibitory effects on osteoblast viability and differentiation, which influences physiological bone turnover, with both excessive osteoclast formation and reduced osteoblast differentiation from mesenchymal stem cells [11].

The present study revealed that at week four, the difference in uric acid level between the hyperuricemic groups and the sham operation group was statistically significant, suggesting that the hyperuricemic model of rats was successfully established. However, the differences in blood calcium, phosphorus, alkaline phosphatase and other bone metabolism markers among groups were not statistically significant. These results reveal that hyperuricemia and androgen did not change the bone metabolism in all groups, and this may be due to the short time of the establishment of the model. Hence, the investigators decided to prolong the modeling establishment time.

At week six, the uric acid level in the hyperuricemic groups also increased, when compared with the sham operation group. But the size of this increase diminished. The level of uric acid decreased in rats in the hyperuricemic groups, when compared with the results in week four. This may 
be correlated with the changes in enzymes and the increase in compensatory excretion in rats. Under this condition, the difference in calcium, phosphorus and alkaline phosphatase between these groups was not statistically significant. However, this hyperuricemic status decreased the calcium level of rats at week six. This confirms the view that hyperuricemia can deteriorate the bone metabolism [16, 26-28]. However, the underlying mechanism remains unknown, at present.

With respect to the correlation between androgen and uric acid, a recent study revealed that the prevalence of hyperuricemia was significantly higher in men than in premenopausal women. That is, androgen increases uric acid levels more effectively than estrogen. The mechanism may be due to the ability of testosterone in androgens to induce the reabsorption of uric acid, allowing the level of uric acid in males to be elevated.

After the adjustment of the modeling protocol, at week eight, the level of uric acid in each group was significantly different from that in the sham operation group. This indicates that the model of hyperuricemia was successful, while the differences in calcium levels between the two castration groups, simple hyperuricemic group and sham operation group were statistically significant. This confirms that androgen has a major effect on bone metabolism in rats.

At week 12, the differences in uric acid levels between the two castration groups and sham operation group were statistically significant. This verifies the effect of androgen on uric acid in non-hyperuricemic rats. That is, androgen increases uric acid levels. This is consistent with a previous research finding [24].

Furthermore, the differences in phosphorus and alkaline phosphatase levels were statistically significant, suggesting the possibility that androgen can decrease bone metabolism in hyperuricemic rats. However, the mechanism of this phenomenon has not been studied in detail. Hence, more experiments are needed to explain the underlying mechanism. Moreover, the present experiment was an animal experiment, and the regulatory mechanism may be different in the inner environment of rats, when compared to humans. Studies with larger sample sizes or further clinical trials are required in the future to explore this mechanism.

In conclusion, androgen can increase serum uric acid levels, and the hyperuricemic status can induce osteoporosis. At the same time, androgen has an effect on bone metabolism under hyperuricemia.

\section{Conflict of interest}

The authors declare no conflict of interest.

\section{References}

1. Yu CX, Zhang XZ, Zhang K, Tang Z. A cross-sectional study for estimation of associations between education level and osteoporosis in a Chinese men sample. BMC Musculoskelet Disord 2015; 16: 382.

2. Jin M, Yang F, Yang I, et al. Uric acid, hyperuricemia and vascular diseases. Front Biosci (Landmark Ed) 2012; 17 : 656-69.

3. Veronese N, Carraro S, Bano G, et al. Hyperuricemia protects against low bone mineral density, osteoporosis and fractures: a systematic review and meta-analysis. Eur J Clin Invest 2016; 46: 920-30.

4. Wauquier F, Leotoing L, Coxam V, Guicheux J, Wittrant Y. Oxidative stress in bone remodelling and disease. Trends Mol Med 2009; 15: 468-77.

5. Wang T, Bi Y, Xu M, et al. Serum uric acid associates with the incidence of type 2 diabetes in a prospective cohort of middle-aged and elderly Chinese. Endocrine 2011; 40: 109-16.

6. Han C, Xian Z, Zou Y, et al. Flow-mediated dilation can be used to predict incident hypertension in patients with hyperuricemia. Arch Med Sci 2019; 15: 343-9.

7. Glantzounis GK, Tsimoyiannis EC, Kappas AM, Galaris DA. Uric acid and oxidative stress. Curr Pharm Des 2005; 11: 4145-51.

8. Ahn SH, Lee SH, Kim BJ, et al. Higher serum uric acid is associated with higher bone mass, lower bone turnover, and lower prevalence of vertebral fracture in healthy postmenopausal women. Osteoporos Int 2013; 24: 2961-70.

9. Zhao DD, Jiao PL, Yu JJ, et al. Higher serum uric acid is associated with higher bone mineral density in chinese men with type 2 diabetes mellitus. Int J Endocrinol 2016; 2016: 2528956.

10. Ahn SH, Lee SH, Kim BJ, et al. Higher serum uric acid is associated withhigherbone mass, lower bone turnover, and lower prevalence of vertebral fracture in healthy postmenopausal women. Osteoporos Int 2013; 24: 2961-70.

11. Chhana A, Callon KE, Pool B, et al. Monosodium urate monohydrate crystals inhibit osteoblast viability and function: implications for development of bone erosion in gout. Ann Rheum Dis 2011; 70: 1684-91.

12. Peng H, Li H, Li C, Chao X, Zhang Q, Zhang Y. Association between vitamin $D$ insufficiency and elevated serum uric acid among middle-aged and elderly Chinese Han women. PLoS One 2013; 8: e61159.

13. Mehta T, Bůžková P, Sarnak MJ, et al. Serum urate levels and the risk of hip fractures: data from the Cardiovascular Health Study. Metabolism 2015; 64: 438-46.

14. Chen W, Roncal-Jimenez C, Lanaspa M, et al. Uric acid suppresses 1 alpha hydroxylase in vitro and in vivo. Metabolism 2014; 63: 150-60.

15. Riggs BL, Jowsey J, Kelly PJ, Jones JD, Maher FT. Effect of sex hormones on bone in primary osteoporosis. J Clin Invest 1969; 48: 1065-72.

16. Bagheri B, Zargari M, Meshkini F, et al. Uric acid and coronary artery disease, two sides of a single coin: a determinant of antioxidant system or a factor in metabolic syndrome. J Clin Diagn Res 2016; 10: OC27-31.

17. Duan X, Ling F. Is uric acid itself a player or a bystander in the pathophysiology of chronic heart failure? Med Hypotheses 2008; 70: 578-81.

18. Sautin YY, Johnson RJ. Uric acid: the oxidant-antioxidant paradox. Nucleosides Nucleotides Nucleic Acids 2008; 27: 608-19.

19. Kurahashi $H$, Watanabe $M$, Sugimoto $M$, et al. Testosterone replacement elevates the serum uric acid levels in 
patients with female to male gender identity disorder. Endocr I 2013; 60: 1321-7.

20. Hernández JL, Olmos JM, González-Macías J. Metabolic syndrome, fractures and gender. Maturitas 2011; 68: 217-23.

21. Wang L, Xu LQ, Shi YF, et al. Establishment of uric acid associated nephropathy animal model and the underlying mechanisms. Chinese Journal of Nephrology 2015; 31: 203-7 [Article in Chinese].

22. Yang GM, Huang SH, Lian XY, Liu Y, Xie YH. Establishment of hyperuricemia model in rats. Laboratory Animal Science 2011; 28: 25-28 [Article in Chinese].

23. Weber HJ. Androgen-estrogen therapy in osteoporosis. Munch Med Wochenschr 1960; 102: 1989-91 [Article in German].

24. Kurahashi $H$, Watanabe $M$, Sugimoto $M$, et al. Testosterone replacement elevates the serum uric acid levels in patients with female to male gender identity disorder. Endocr I 2013; 60: 1321-7.

25. Li RS, Li WJ, Wang Y, Chen HS, Tu Q. The relationship between blood uric acid levels and bone mineral density in people of Liuzhou city. Med Innov China 2016; 13: 138-140, 141 [Article in Chinese].

26. Zheng Y, Zheng FP, Li H. The relationship between serum uric acid level and lumbar bone mineral density in patients with Graves disease. Chinese Journal of Gerontology 2014; 34: 3017-9 [Article in Chinese].

27. Cheng Y, You ZQ, Ai ZH, Wan Y, Guo W. Study on the relationship between bone mineral density and blood uric acid metabolism in middle-aged and old male type 2 diabetic patients. China Journal of Modern Medicine 2015; 25: 37-41 [Article in Chinese]

28. Zhao N, Li SM, Fang XX, et al. Correlation of bone mineral density and blood uric acid level in elderly patients with diabetes. Chinese Journal of Osteoporosis 2014; 20: 784-8 [Article in Chinese] 